\title{
Aerodynamic measures of patients with tracheostomy capping before and after decannulation
}

\author{
Gamal Youssef ${ }^{1,2^{*}}$ (D), Kamal M. Abdulla ${ }^{2}$ and Sahar S. Khalii ${ }^{3,4}$
}

\begin{abstract}
Background: Gradually stepped decannulation, such as capping with fenestrated tubes or tube downsizing, is likely to prolong the decannulation process. The purpose of the study is to determine the differences in laryngeal aerodynamic measures, expiratory peak flow, and dyspnea index when breathing through the upper airway before and after decannulation. The study recruited sixteen adult patients with a tracheostomy who were fit for decannulation. Measurement of peak flow rate, aerodynamic measures, and dyspnea index has been done at two settings: first during capping and second after decannulation by $2 \mathrm{~h}$ using finger support to close the stoma and prevent air leak.

Results: Changes in outcome measures after decannulation revealed a significant increase in peak flow rate, vital capacity, maximum phonation, and phonatory speech pressure level; the glottal resistance significantly decreased after decannulation. The dyspnea severity index scores improved from 22.35 during capping to 13.37 after decannulation.
\end{abstract}

Conclusions: The results of our study showed that tracheostomy tube capping causes a significant reduction in peak flow and aerodynamic measures which improved after tracheostomy decannulation.

Keywords: Tracheostomy, Decannulation, Aerodynamic, Capping, Downsizing

\section{Background}

Tracheostomy decannulation decision is the major challenge in the clinical management of tracheostomy patients. Little evidence is available to guide the weaning process and optimal timing of tracheostomy tube removal. Several protocols for tracheostomy decannulation have been described in the literature. The most used technique is tube capping for $48 \mathrm{~h}$ with a gradual downsizing of the tube or using a fenestrated tube; however, this method is subjective which depends on the patient's impressions and clinical judgments. When the tube size reduced, the airflow becomes turbulent and airway resistance increased; this makes the patient prone to more

\footnotetext{
*Correspondence: dr.gamal@gmail.com; gyyoussef@dha.gov.ae

${ }^{2}$ ENT Department, Dubai Hospital, Dubai Health Authority (DHA), Abu

Bakr Al Siddiq street, Dubai, United Arab Emirates

Full list of author information is available at the end of the article
}

resistance when breathing through a smaller diameter. As the resistance is inversely related to the tube diameter, it raised from the 4th to the 5th power when the laminar flow changed to turbulent $[1,2]$.

Intermediate step of decannulation, as tube capping with a fenestrated tube, depends on noninvasive checking of the upper airway patency. It starts by finger closure of the tracheostomy to redirect air through the vocal folds and upper airway, allowing breathing and phonation. If the patient passes finger occlusion test, then gradual tube capping starts and the patient is observed for any signs of respiratory distress. Gradually stepped decannulation such as capping with the use of fenestrated tubes or tube downsizing is likely to prolong the decannulation process [1]. Endoscopic visualization of the laryngeal and tracheal airway has been advised to be an objective protocol before decannulation. The endoscopic protocol in tracheostomized patients could improve the tracheostomy 
decannulation decisions and the rate of success of decannulation.

Tracheostomy decannulation is necessary to improve patient security, perceived appearance, swallowing, and vocal folds functions that lead to improved independence, social interaction, and inclusion [3].

In the clinical practice of tracheostomy decannulation, some patients feel difficulty in breathing during a gradual decannulation trial using fenestrated tube capping; however, some of these patients feel comfortable breathing after decannulation. This type of study has not been addressed previously. These findings lead us to hypothesize that the tracheostomy tube itself may affect airway resistance, easiness of breathing, and laryngeal aerodynamics during tube capping.

Since most clinical evaluations are based on pre-decannulation measures only, the ability to predict post-decannulation respiratory and phonatory ability is important in assessing a patient's candidacy for decannulation $[4,5]$.

Many studies examine the difference between mouth breathing and tracheal breathing in tracheostomized patients. To the best of our knowledge, no previous studies have compared aerodynamic measures in patients with capped fenestrated tracheostomy before and after decannulation. Several studies had recommended analysis of a patient with difficult decannulation with modification in the tracheostomy decannulation protocol, and they investigated the factor that could predict successful decannulation, to minimize the risk of respiratory breakdown [6-8].

The study aimed to determine the differences in laryngeal aerodynamic measures, expiratory peak flow, and dyspnea index when breathing through the upper airway with an occluded cuffless fenestrated tracheostomy tube, and after decannulation. Furthermore to highlight the role of decannulation in improving those parameters that could improve confidence in decannulation decisions.

\section{Methods}

This cross-sectional study was conducted in the ENT department, Dubai hospital, in the period between February 2019 and January 2020. The study included sixteen adult patients with a tracheostomy who were fit for decannulation according to our institutional decannulation protocol [9]. Seven patients were due to prolonged mechanical ventilation, four patients were due to head and neck surgery, and five patients were due to cerebrovascular stroke. The protocol of endoscopic tracheostomy assessment included fiberoptic endoscopic evaluation of swallowing (FEES), with additional of endoscopic subglottic and tracheal airway assessment. The results of the endoscopic assessment were categorized according to fitness for decannulation decision either fit or unfit for decannulation.

We established the following criteria for inclusion in the study: adult tracheostomized patient, patients were free from upper airway obstruction, can manage his saliva, has a good cough, and can tolerate cuff deflation with spontaneous breathing in room air.

We excluded individuals who could not cooperate in aerodynamic measuring or responding to the dyspnea index (DI) questionnaire due to cognitive disorders and those who did not meet any of the inclusion criteria.

We applied the routine gradual weaning approach using a fenestrated tube with an inner diameter median was $7.0 \mathrm{~mm}$ (range, $6.0-7.5 \mathrm{~mm}$ ). Capping of tracheostomy tube was done $48 \mathrm{~h}$ with $\mathrm{PO} 2$ monitoring before final decannulation and stoma closure.

Evaluation and measurement were done at two settings: first during capping and second after decannulation by $2 \mathrm{~h}$ with finger support to close the stoma to prevent air leak.

\section{Outcome measures:}

- Peak flow rate (PFR): the patient was instructed to exhale as forcefully as possible via a peak flow meter (Findel, England) at the mouth.

- Maximum phonation time (MPT): The patient instructed to phonate as maximum as possible on a single breath to measure the MPT in seconds as the highest of three attempts.

- Aerodynamic measures of the larynx: using Aerophone II (Model 6800 - Kay Pentax., USA). The patient was asked to hold the mask firmly around his mouth and hold an intraoral tube between lips to measure mean subglottic pressure (Psub), glottal resistance, and phonatory speech pressure level (SPL). The vital capacity (VC) measured as the amount of air can exhale after maximum inhalation and the phonatory Quotient (PQ) calculated as the ratio of VC to MPT.

- Dyspnea index (DI): it is a validated clinical assessment tool to assess airway disease burden and effect on the quality of life. It includes ten questions on a 5-point Likert scale with a total score of 40 (e.g., "I have trouble getting air in"; 0: never; 1: almost never; 2: sometimes; 3: almost always; and 4: always). DI was collected for all patients one day before decannulation during capping and post decannulation [10].

\section{Statistical analysis}

Statistical analyses were performed with SPSS 20 (Statistical Package for Social Sciences) (SPSS Inc. Chicago, IL, 
USA). Descriptive statistics were expressed as frequency and percentage for nominal variables and mean and standard deviation for numerical variables. The data were tested for normal distribution using Shapiro-Wilk test. In normally distributed data, we used parametric test as paired sample $t$ test to study significance between preand post-decannulation measures. The related samples Wilcoxon signed-rank test were used for non-normally distributed data. A two-sided $p<0.05$ were considered as statistically significant.

\section{Results}

The mean age of the studied group was $50.6+16.8$ and range from 28 to 68 years. The sex distribution was 11 (68.8\%) males and 5 (31.2) females. Eleven patients had a surgical tracheostomy and five patients underwent a percutaneous tracheostomy. Of the 16 patients enrolled, seven patients were due to prolonged mechanical ventilation, four patients were due to head and neck surgery, and five patients were due to cerebrovascular stroke. The range of duration of tracheostomy till decannulation was from 21 to 85 days, with mean $38+32$ days. All patients succeeded in the decannulation process.

\section{Changes in outcome measures after decannulation:}

- The dyspnea index scores mean has improved from 22.35 during capping to 13.37 after decannulation (mean of difference, $-8.7 ; 95 \% \mathrm{CI},-11.5$ to -6.2 ); the improvement was statistically significant as shown in Table 1 and Fig. 1.

- Peak flow rate has significantly increased after decannulation in most patients, as shown in Fig. 2.

- Aerodynamic measures: Table 1 and Figs. 3, 4, 5, and 6 show the differences in all outcome measures before and after decannulation. It revealed a significant increase in vital capacity, maximum phonation, and phonatory speech pressure level; though, the glottal resistance has significantly decreased after decannulation. The changes in the subglottic pressure and phonatory quotient were not statistically significant.

\section{Discussion}

Until date, there is no consensus regarding the ideal method of tracheostomy decannulation, leaving the decision to the institutional guidelines and expert opinions. A successful decannulation requires the patient to ventilate through the natural upper airway and to tolerate occluded or capped fenestrated cuffless tube. If that process is conducted prematurely or without proper observations, it could lead to severe fatal complications $[7,11]$. While most studies compared mouth breathing through a fenestrated tube and breathing through a tracheostomy, our study compared differences between mouth breathing through a capped fenestrated tube and mouth breathing after decannulation. No previous reports have described the effects of the tracheostomy tube capping on breathing and laryngeal aerodynamics measures [12]. We use the fenestrated cuffless tube as the airway flow resistance with a capped tracheostomy tube with deflated cuff is higher than that with capped fenestrated cuffless tube [13]. This study demonstrated significant differences in most studied aerodynamic parameters before and after decannulation. There were significant increases in peak flow rate, vital capacity, maximum phonation time, and SPL after decannulation.

The changes in aerodynamic measures are attributed to many factors. The larynx contributes a small fraction of the overall airflow resistance in normal circumstances and work of breathing is the inspiratory step of the respiratory cycle [1]. Phonatory aerodynamic are expiratory functions that depend on the interaction between airflow and glottal airway resistance. The relationship between

Table 1 The differences in the outcome measures before and after decannulation

\begin{tabular}{|c|c|c|c|c|c|c|}
\hline \multirow[t]{2}{*}{ Variable } & \multicolumn{2}{|c|}{ Pre-decannulation } & \multicolumn{2}{|c|}{ Post-decannulation } & \multirow[t]{2}{*}{$T / Z$ value } & \multirow[t]{2}{*}{$P$ significance } \\
\hline & Mean (SD) & Range & Mean (SD) & Range & & \\
\hline Dyspnea Index (0-40) & $22.25(4.80)$ & $12-32$ & $13.37(4.55)$ & $8-25$ & 7.150 & .000 \\
\hline $\operatorname{PFR}(\mathrm{L} / \mathrm{m})$ & $2.76(0.57)$ & $1.90-3.70$ & $3.12(0.48)$ & $2.40-3.80$ & -5.461 & .000 \\
\hline MPT (seconds) & $10.3(2.7)$ & $6.5-15.3$ & $12.1(3.1)$ & $6.2-16.7$ & -2.664 & .018 \\
\hline VC (liters) & $2.72(0.82)$ & $1.62-4.20$ & $3.01(.80)$ & $1.90-4.70$ & -5.030 & .000 \\
\hline$P Q, L / s$ & $0.277(0.095)$ & $0.15-0.46)$ & $0.255(.056)$ & $0.18-0.37$ & 1.154 & .266 \\
\hline Psub, $\mathrm{CmH} 2 \mathrm{O}$ & $7.09(0.85)$ & $5.60-8.50$ & $6.91(1.95)$ & $4.20-11.24$ & .448 & .661 \\
\hline Pspl, dB & $67.62(6.37)$ & $56.3-77.8$ & $75.12(5.74)$ & $48.6-84.7$ & -5.165 & .000 \\
\hline $\mathrm{GR}, \mathrm{CmH} 2 \mathrm{O} / \mathrm{L} / \mathrm{s}$ & 88.84 (25.92) & $47.5-148.2$ & $74.89(20.63)$ & $35.5-124.5$ & 4.232 & .001 \\
\hline
\end{tabular}




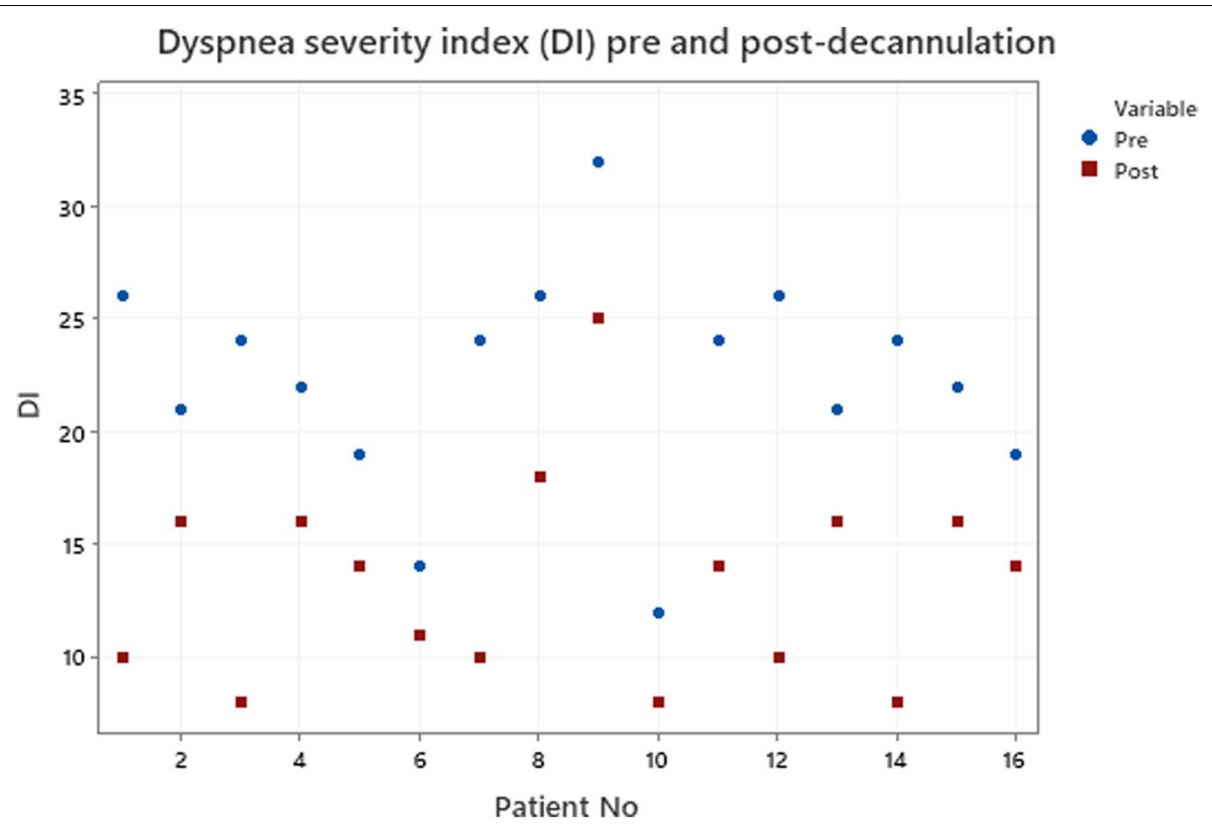

Fig. 1 Plot distribution of dyspnea severity index in all studied subjects before and after decannulation

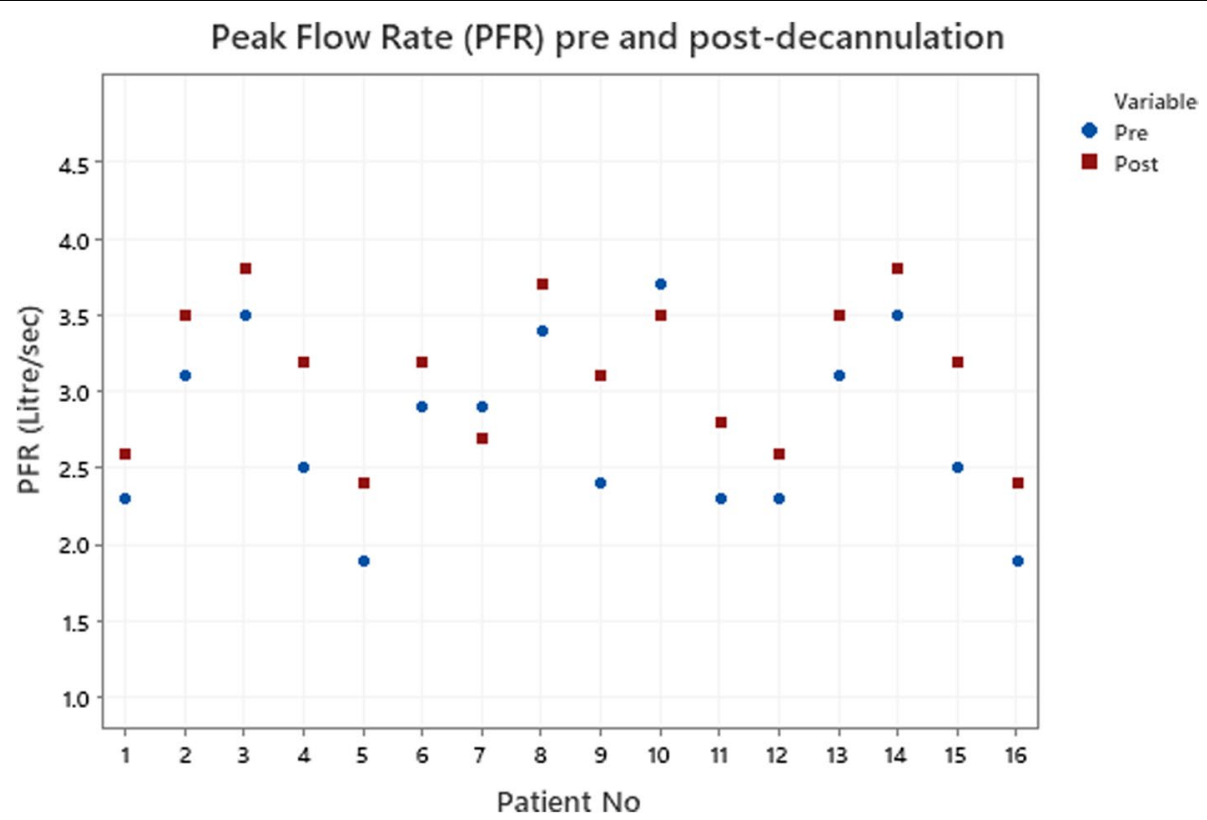

Fig. 2 Plot distribution of peak flow rate in all studied subjects before and after decannulation

breathing and voice are essential as any obstruction in the airway leads to loss of the laryngeal fine-tuning capabilities. A rise in the respiratory effort makes it difficult to sustain adequate speech pressure level [14]. There is an association between the volume of the lung and subglottic pressure (Psub), and Psub is greater at high lung volume than at low volume. The insufficient airflow to promote the Bernoulli's effect during the glottic cycle leads to altered SPL, Psub, and MPT observed in our study as predicted by the aerodynamic-myoelastic theory [15].

According to our findings, we can admit that tracheostomy tube capping causes an apparent airflow obstruction which should be considered during tracheostomy 


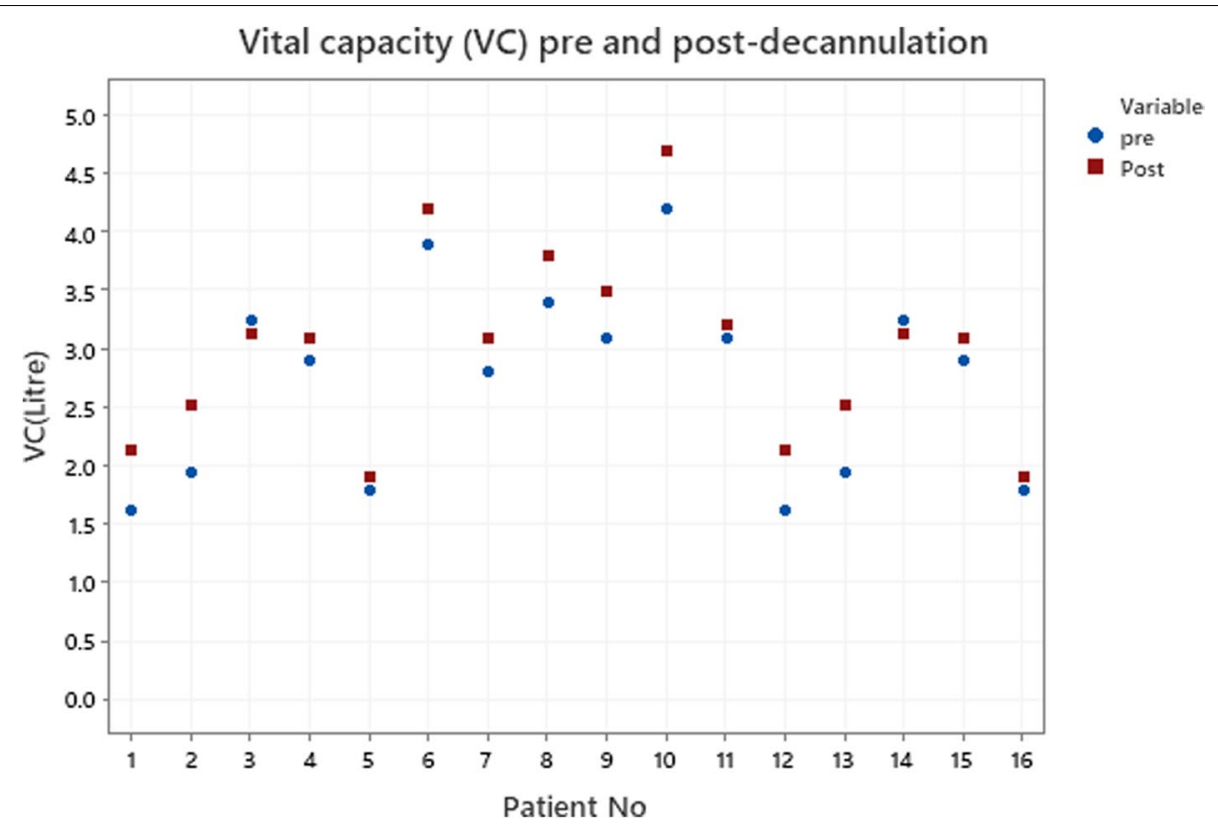

Fig. 3 Plot distribution of vital capacity in all studied subjects before and after decannulation

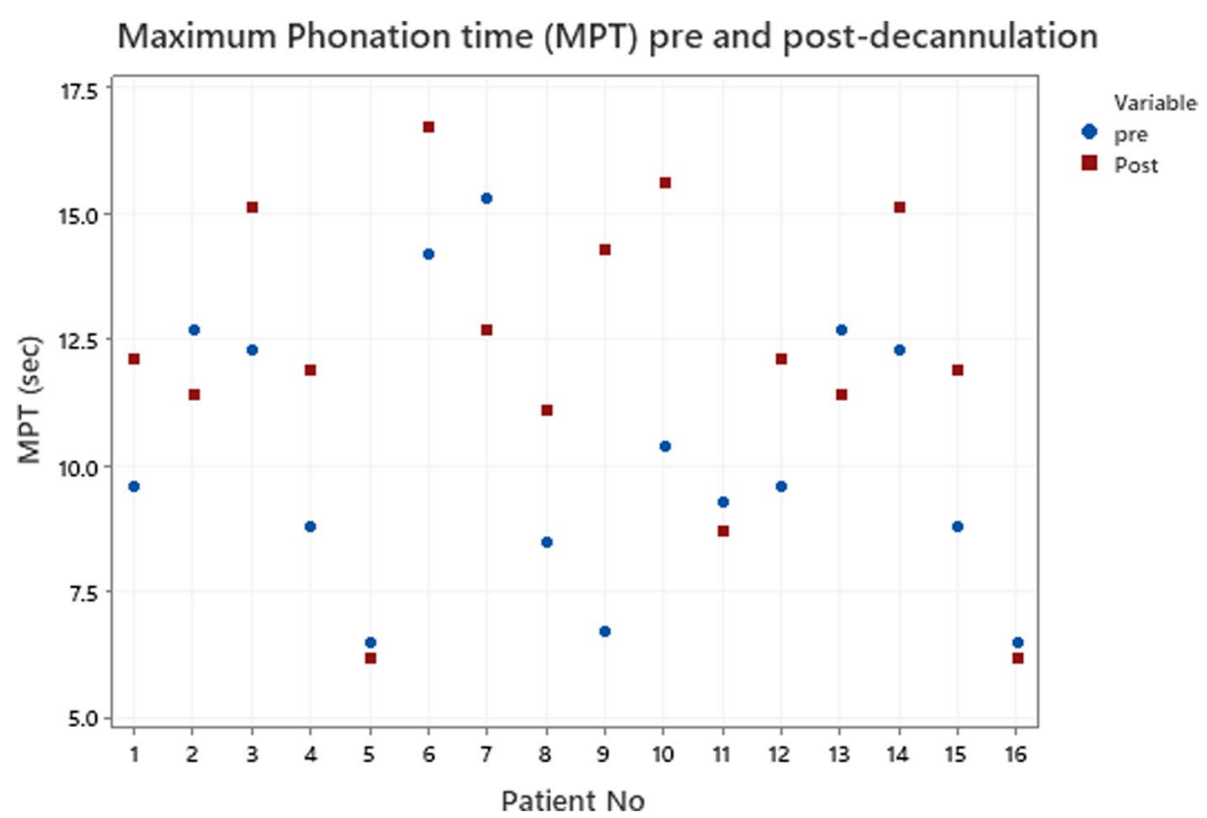

Fig. 4 Plot distribution of maximum phonation time in all studied subjects before and after decannulation

capping trials. These findings are in agreement with the recent study on tracheostomy patients with an occluded fenestrated cuffless tube. They used a portable spirometer to measure vital capacity, forced expiratory volume, and peak flow rate in two settings before and immediately after decannulation through mouth breathing. They found a significant increase of studied respirometer parameters after decannulation and concluded that capped tracheostomy causes a remarkable airflow obstruction, mostly in the expiratory phase [16].

The work of breathing increases when breathing through the mouth with an occluded tracheostomy tube than after decannulation. Fenestrated tube worsens resistance to exercise, airflow resistance, and muscle 


\section{Phonatory speech pressure level (PSPL) pre and post-decannulation}

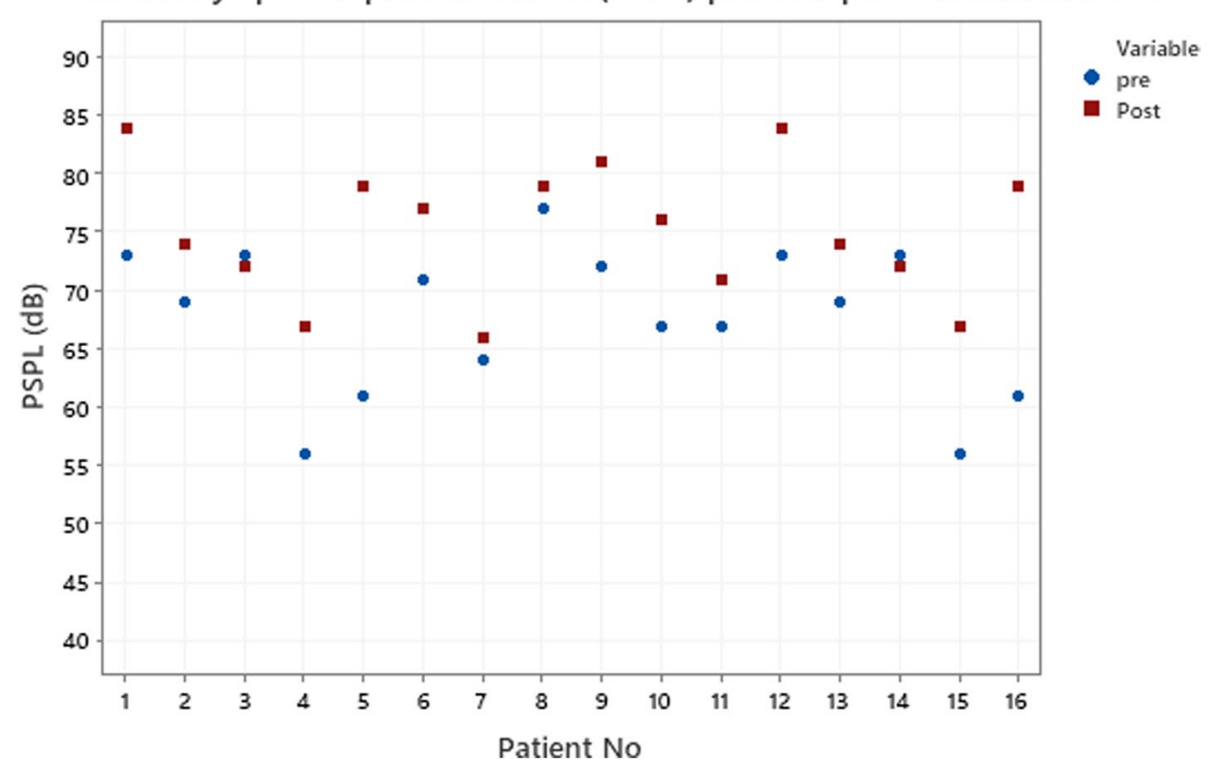

Fig. 5 Plot distribution of phonatory speech pressure level in all studied subjects before and after decannulation

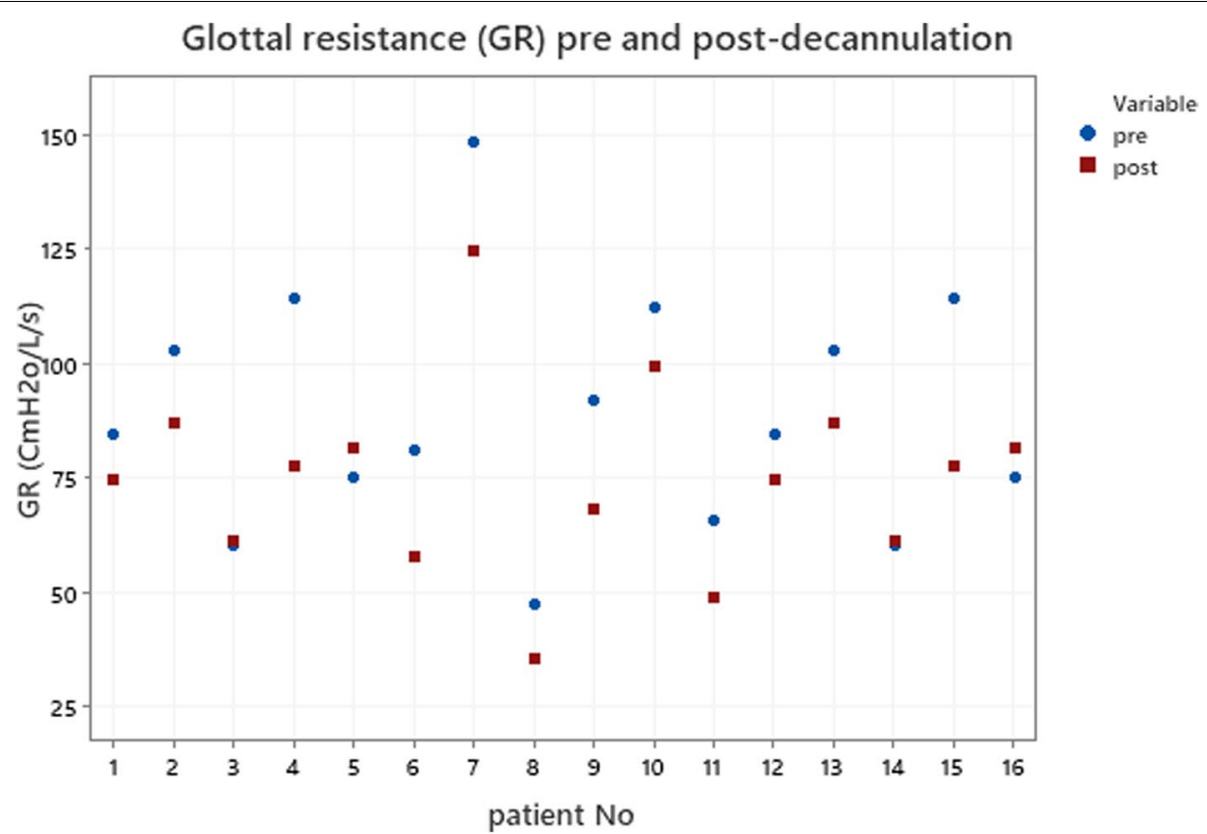

Fig. 6 Plot distribution of glottal resistance in all studied subjects before and after decannulation

performance. This may be related to generating more resistance by decreasing the inner diameter of the trachea due to the presence of the tube body in the trachea. Those changes need special consideration in tracheostomy decannulation in patients with pulmonary or neurological comorbidities $[6,12]$.
A previous study suggested a minimum peak inspiratory flow (PIF) of $40 \mathrm{l} / \mathrm{min}$ as a simple tool for prediction of decannulation success and they used upper airway endoscopy only in cases of repeated failed decannulations. They suggested not to cap the tracheostomy tube before decannulation for the reason that the existence of 
the tube in the trachea disturbs coughing and diminishes airway diameter [5].

In comparing cough peak flow (CPF) measured from the mouth before and after decannulation in a previous investigation. They concluded that post-decannulation CPF could be predicted to increase by about $34 \mathrm{l} / \mathrm{min}$ greater than the pre-decannulation values, and this finding lowers the threshold level of CPF indicated for safe decannulation $[17,18]$.

Previous research concluded that airway resistance was the best predictor for post-decannulation alteration in the work of breathing [19]. Unlike our study, that study did not measure mouth breathing with and without tracheostomy tube, so it did not study the effect of tracheostomy tube on airflow and upper airway resistance as the present study found.

Another study used a stoma stent as an intervening step in high-risk tracheostomy decannulation. The stoma stent advantage over the capped tracheostomy tube could be the unobstructed tracheal lumen with patent trachea and easier secretion clearance via coughing. The upper airway resistance and vocal folds dysfunction can fluctuate over time due to fatigue, tension, difference in anatomy, patient position, sticky secretions, and tracheostomy tube airway complications. Speech pathology identification and treatment of vocal folds dysfunction may improve patients' performance through vocal hygiene [20].

In the present study, we use the dyspnea index as a patient subjective feeling of breath comfort, which indicates better ease of breath after decannulation in most patients. That point is essential because patient discomfort in the capping process can postpone decannulation, so decannulation has to be tried with readiness to re-cannulate if discomfort does not improve. All our studied patients have tolerated capping despite decrease flow rate which improved after decannulation. The previous study on tracheostomy capping protocol found that $16 \%$ of patients developed signs of respiratory distress and failure of capping due to airflow obstruction [21]. In the current study, we use an endoscopic assessment that excluded obstructive upper airway lesion so the success rate was $100 \%$. For patients who did not breathe for several weeks through the upper airway, they have fear about the different breathing patterns, resistance, and sensation. This phenomenon should be clinically differentiated from distress due to suspected upper-airway obstruction [1].

Our findings highlight the importance of endoscopic airway assessment to exclude any airway obstruction before capping, which improves the clinician's confidence in decannulation decisions [9]. One of the recent systemic reviews recommended changing from the fixed protocolized approach of decannulation to a more flexible individualized approach for decannulation built on validated methods [22].

\section{Conclusions}

The results of our study showed that tracheostomy tube capping causes a significant deterioration in peak flow, aerodynamic measures, and patient comfort of breathing which improve after tracheostomy decannulation. Our findings highlight the importance of endoscopic airway assessment to exclude any airway obstruction before capping which improves the clinician confidence in decannulation decisions.

\section{Abbreviations}

PFR: Peak flow rate; MPT: Maximum phonation time; Psub: subglottic pressure; GR: Glottal resistance; pSPL: Phonatory speech pressure level; VC: The vital capacity; PQ: Phonatory quotient; DI: Dyspnea index.

\section{Acknowledgements}

Nil.

\section{Authors' contributions}

All authors have contributed to designing the study; collecting, analyzing, and interpretation of data; and preparing and revising the manuscript. Design of the study: GY. Recruitment of patients: GY and KA. Data collection: GY, KA, and SK. Statistical analysis and data interpretation: GY and SK. Manuscript preparation: GY, KA, and SK. Manuscript revision: GY and SS. All authors have approved the final version of the manuscript.

Funding

Nil.

\section{Availability of data and materials}

The datasets during and/or analyzed during the current study are available from the corresponding author on reasonable request.

\section{Declarations}

Ethics approval and consent to participate

Ethics committee approval obtained from clinical governance office in Dubai Health Authority with reference number. DSRC-09/2019-10.

Informal consent to participate was obtained from the patients before starting the study.

\section{Consent for publication}

Not applicable.

\section{Competing interests}

The authors declare that they have no competing interests.

\section{Author details}

${ }^{1}$ Phoniatrics Unit, ENT Department, Faculty of Medicine, Alexandria University, Alexandria, Egypt. ${ }^{2}$ ENT Department, Dubai Hospital, Dubai Health Authority (DHA), Abu Bakr Al Siddiq street, Dubai, United Arab Emirates. ${ }^{3}$ Rhumatology and Rehabilitation Department, Faculty of Medicine, Zagazig University, Zagazig, Egypt. ${ }^{4}$ PMR department, Dubai Hospital, Dubai Health Authority (DHA), Dubai, United Arab Emirates.

Received: 2 November 2021 Accepted: 31 December 2021 Published online: 21 January 2022 


\section{References}

1. Cohen O, Tzelnick S, Lahav Y, Stavi D, Shoffel-Havakuk H, Hain M, Halperin D, Adi N (2016) Feasibility of a single-stage tracheostomy decannulation protocol with endoscopy in adult patients. Laryngoscope 126(9):2057-2062

2. Carter A, Fletcher SJ, Tuffin R (2013) The effect of inner tube placement on resistance and work of breathing through tracheostomy tubes: a bench test. Anaesthesia. 68:276-282

3. McKim DA, Hendin A, LeBlanc C, King J, Brown CRL, Woolnough A (2012) Tracheostomy decannulation and cough peak flows in patients with neuromuscular weakness. Am J Phys Med Rehabil 91:666Y670

4. Singh RK, Saran S, Baronia AK (2017) The practice of tracheostomy decannulation - a systematic review. J Intensive Care 5(1):38

5. Guerlain J, Guerrero JA, Baujat B et al (2015) Peak inspiratory flow is a simple means of predicting decannulation success following head and neck cancer surgery: a prospective study of fifty-six patients. Laryngoscope 125:365-370

6. Chadda K, Louis B, Benaïssa L, Annane D, Gajdos P, Raphaël J, Lofaso $F$ (2002) Physiological effects of decannulation in tracheostomized patients. Intensive Care Med 28(12):1761-1767

7. Pandian V, Miller CR, Schiavi AJ, Yarmus L, Contractor A, Haut ER, FellerKopman DJ, Mirski MA, Morad AH, Carey JP, Hillel AT (2014) Utilization of a standardized tracheostomy capping and decannulation protocol to improve patient safety. Laryngoscope 124(8):1794-1800

8. Morris LL, Mcintosh E, Whitmer A (2014) The importance of tracheostomy progression in the intensive care unit. Crit Care Nurse 34(1):40-48

9. Youssef G, Abdulla KM (2020) Value of endoscopic examination of airways and swallowing in tracheostomy decannulation. Egypt J Otolaryngol 36:1-6

10. Gartner-Schmidt JL, Shembel AC, Zullo TG, Rosen C (2014) Development and validation of the Dyspnea Index (DI): a severity index for upper airway-related dyspnea. J Voice 28(6):775-782

11. Christopher KL (2005) Tracheostomy decannulation. Respir Care 50(4):538-541

12. Villalba D, Feld V, Leiva $V$ et al (2016) Effect of tracheostomy tube on work of breathing: Comparison of pre- and post-decannulation. Int J Crit IIIn Inj Sci 6(3):98-102. https://doi.org/10.4103/2229-5151.190651

13. Hussey JD, Bishop MJ (1996) Pressures required to move gas through the native airway in the presence of a fenestrated vs a nonfenestrated tracheostomy tube. Chest 110:494-497

14. Lewandowski A, Gillespie Al (2016) The relationship between voice and breathing in the assessment and treatment of voice disorders. Perspect ASHA Special Inter Groups 1(3):94-104

15. Iwarsson J, Thomasson M, Sundberg J (1998) Effects of lung volume on the glottal voice source. J Voice 12(4):424-433.)

16. Sanchez-Guerrero JA, Guerlain J, Cebrià i Iranzo MÀ, Baujat B, Lacau St Guily J, Périé S (2020) Expiratory airflow obstruction due to tracheostomy tube: a spirometric study in 50 patients. Clin Otolaryngol 45(5):703-709

17. McKim DA, Hendin A, LeBlanc C, King J, Brown CR, Woolnough A (2012) Tracheostomy decannulation and cough peak flows in patients with neuromuscular weakness. Am J Phys Med Rehabil 91(8):666-670

18. Park MK, Lee SJ (2012) Changes in Swallowing and Cough Functions Among Stroke Patients Before and After Tracheostomy Decannulation. Dysphagia 33(6):857-865

19. Dellweg D, Barchfeld T, Haidl P, Appelhans P, Kohler D (2007) Tracheostomy decannulation: implication on respiratory mechanics. Head Neck 29(12):1121-1127

20. Ross J, McMurray K, Cameron T, Lanteri C (2019) Use of a silicon stoma stent as an interim step in high-risk tracheostomy decannulation. OTO Open 3(1):2473974X19836432

21. Rumbak MJ, Graves AE, Scott MP, Sporn GK, Walsh FW, Anderson WM, Goldman AL (1997) Tracheostomy tube occlusion protocol predicts significant tracheal obstruction to air flow in patients requiring prolonged mechanical ventilation. Crit Care Med 25(3):413-417

22. Kutsukutsa J, Kuupiel D, Monori-Kiss A, Del Rey-Puech P, MashambaThompson TP (2019) Tracheostomy decannulation methods and procedures for assessing readiness for decannulation in adults: a systematic scoping review. Int J Evid-based Healthcare 17(2):74-91

\section{Publisher's Note}

Springer Nature remains neutral with regard to jurisdictional claims in published maps and institutional affiliations.

\section{Submit your manuscript to a SpringerOpen ${ }^{\circ}$ journal and benefit from:}

- Convenient online submission

- Rigorous peer review

- Open access: articles freely available online

- High visibility within the field

- Retaining the copyright to your article

Submit your next manuscript at $\boldsymbol{\nabla}$ springeropen.com 